\title{
. Update on the management of atrial fibrillation
}

John V Amerena MB BS, FRACP, FCSANZ, Director of Cardiology Research,' and Associate Professor of Medicine

Tomos E Walters MB BS

Electrophysiology Fellow

Sam Mirzaee

Cardiology Research Fellow

Jonathan M Kalman MB BS, PhD, FRACP.

Director of Cardiac Electrophysiology, ${ }^{3}$ and Professor of Medicine

1 Geelong Cardiolog ResearchUnit, Geelong, VIC.

2 Deakin University, Geelong, VIC

3 Department of Cardiology, Royal

Melbourne Hospital, Melbourne, VIC

4 Barwon Health Geelong, VIC

5 University of Melbourne, Melbourne, VIC

johnam@

barwonhealth.org.au

MJA 2013; 199: 592-597 doi: $10.5694 / \mathrm{mjal3.1019}$

Previously in this series "Cardiology series - 4" in MJA 2013; 199: 334-339

Series Editors Derek P B Chew MB BS, MPH, FRACP Ian A Scott MBBS, FRACP, MHA trial fibrillation $(\mathrm{AF})$ is the most common cardiac rhythm disorder in clinical practice, with a prevalence $<1 \%$ in people aged under 60 years and increasing to $10 \%$ in those older than 80 years. ${ }^{1-3}$ AF can markedly impair quality of life through symptoms such as palpitations, fatigue and dyspnoea and is independently associated with an increased risk of stroke, heart failure and death. ${ }^{4-6}$ With an increasing incidence of AF and an ageing population in the developed world, the implications for health care costs are significant.

AF may be classified as paroxysmal, with self-terminating episodes of $\leqslant 48$ hours, or as persistent, in which episodes last longer than 7 days or require cardioversion to restore sinus rhythm. ${ }^{7}$ Longstanding persistent $\mathrm{AF}$ is defined as lasting longer than 1 year, and permanent $\mathrm{AF}$ as being refractory to cardioversion or when attempts at establishing sinus rhythm are no longer pursued.

Here, we provide a practical review of the issues faced in managing patients with $\mathrm{AF}$, including considerations in deciding between rate control and rhythm control approaches, and the role of catheter ablation. As stroke prevention remains paramount, we discuss the use of warfarin and the new oral anticoagulant (NOAC) drugs.

\section{Diagnostic considerations}

AF is typically identified by fibrillatory waves on an electrocardiogram, with an "irregularly irregular" ventricular response. In patients with intermittent symptoms, the use of Holter monitors, event recorders or implantable loop recorders may be needed to confirm the diagnosis.

Secondary causes of AF need to be excluded, and specific tests in individual patients might include thyroid function tests, measurement of ambulatory blood pressure, and sleep studies to detect sleep apnoea. Echocardiography should be routinely performed to look for associated structural heart disease, and coronary disease should be excluded in patients with suggestive symptoms. With a growing array of anticoagulant options and an increase in the use of AF ablation, ${ }^{8}$ all patients with newly diagnosed $\mathrm{AF}$, but particularly those who are younger (ie, aged $<65$ years) and with more severe symptoms, should be considered for specialist referral.

AF is commonly associated with cardiovascular comorbidities such as valvular heart disease, hypertension, diabetes mellitus, left ventricular dysfunction and vascular disease. ${ }^{9,10}$ In recent years, increasing attention has been given to the AF risk conferred by obesity and obstructive

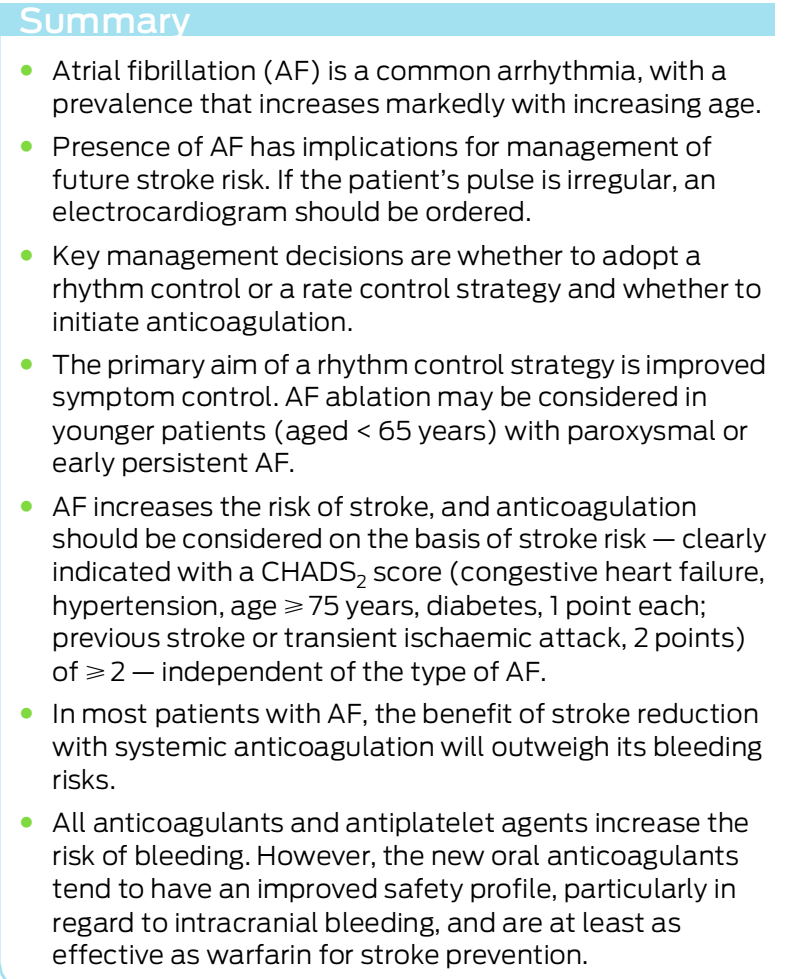

sleep apnoea (OSA). Obesity (body mass index $>30 \mathrm{~kg} / \mathrm{m}^{2}$ ) is a key component of the metabolic syndrome and has now been demonstrated to increase risk of $\mathrm{AF}$ independent of other risk factors. ${ }^{11-14}$ Left atrial enlargement associated with diastolic dysfunction has been identified as a key intermediary. The presence of pericardial fat - a metabolically active ectopic fat deposit in direct contact with, and sharing a microcirculation with, cardiac tissue - has been specifically implicated in the pathogenesis of $\mathrm{AF}$, independent of measures of systemic adiposity. ${ }^{15,16}$ OSA is characterised by periodic obstruction of the upper airways and apnoea. Periods of hypoxaemia and hypercapnia, with repeated arousals from sleep, lead to sympathetic activation and elevated blood pressure. AF risk is increased in patients with OSA and is related to OSA severity, $7,17-19$ independent of cardiac disease or other comorbidities. AF recurrence after cardioversion is also more common in patients with OSA.

In the Atherosclerosis Risk in Communities (ARIC) study, $57 \%$ of patients presenting with incident $\mathrm{AF}$ had poor control of at least one cardiovascular risk factor. ${ }^{20}$ 
However, AF can be seen in younger patients with no evidence of comorbid cardiopulmonary disease - this condition is termed "lone $\mathrm{AF}^{\prime}$ and has been identified in $1.6 \%$ to $30 \%$ of patients. ${ }^{21}$ Given the absence of comorbidities, it carries a low risk of stroke.

\section{Management}

\section{Rhythm control or rate control}

A key issue in managing $\mathrm{AF}$ is to decide between attempting to maintain or restore sinus rhythm or to accept $\mathrm{AF}$ and control the ventricular rate (Box 1). This should not be an immutable decision, as AF is a dynamic condition in which symptom status, stroke risk and even patient desires and expectations may evolve.

Randomised trials have not demonstrated a reduction in mortality or thromboembolism with rhythm control, but these studies have frequently included older people with structural heart disease, in whom the success of rhythm control has been poor. ${ }^{22-27}$ A recent meta-analysis showed no significant difference between rate and rhythm control for all-cause mortality, cardiovascular mortality or ischaemic stroke in patients with $\mathrm{AF}^{28}$ Post-hoc analyses have suggested that outcomes are improved for those patients in whom sinus rhythm can be effectively maintained, and there is no doubt that many patients with $\mathrm{AF}$ derive significant symptomatic benefit from achieving sinus rhythm. Rhythm control remains the first-line option in symptomatic patients without advanced structural cardiac disease, particularly those without significant left atrial enlargement, which renders long-term maintenance of sinus rhythm more challenging.

For ventricular rate control, atrioventricular nodal blocking agents include $\beta$ blockers, calcium channel blockers (verapamil, diltiazem) and digoxin (Box 2). Symptom control and prevention of left ventricular systolic dysfunction, which can develop with sustained tachycardia, is the usual
1 Factors influencing a decision between rhythm control or rate control for atrial fibrillation (AF)
Factors favouring a rhythm control approach:

- Younger age

- More frequent AF episodes associated with more severe symptoms

- Heart failure triggered by AF

- No more than moderate left atrial enlargement

- No contraindications to antiarrhythmic drugs association with cardioversion
- Acceptance of anticoagulation in
Factors favouring a rate control approach:

- Advanced age

- Longstanding persistent AF

- Severe left atrial enlargement

- Multiple previous attempts to restore sinus rhythm

- AF with minimal associated symptoms

- Non-AF comorbidities with greater impact on overall quality of life

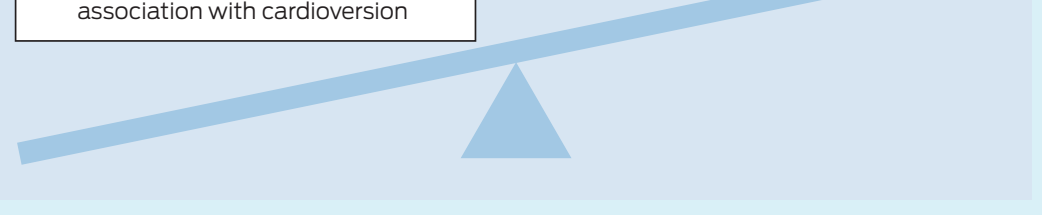

clinical approach, rather than achieving a specific target heart rate. For patients with refractory symptoms, a 24hour Holter monitor is useful to assess response to therapy during daily activities. An average heart rate of $<100$ beats/ min provides a clinically used indicator of acceptable rate control, although in the Rate Control Efficacy in Permanent Atrial Fibrillation: a Comparison between Lenient versus Strict Rate Control II (RACE II) study, there was no excess of adverse outcomes associated with lenient rate control (resting heart rate, $<110$ beats $/ \mathrm{min}$ ) compared with more strict control (resting heart rate, $<80$ beats/min; heart rate during exercise, $<110$ beats $/ \mathrm{min}){ }^{29}$

Rhythm control involves a combination of electrical cardioversion and pharmacological therapy to achieve and maintain sinus rhythm. Rhythm control drugs act by alter-

\section{Rate control and antiarrhythmic drugs commonly used for the management of atrial fibrillation (AF) in Australia*}

Drug

\section{Adverse effects}

Rate control drugs

$\beta$ Blockers

Bradycardia, heart block, bronchospasm

Calcium channel blockers

(non-dihydropyridine)

Digoxin

\section{Rhythm control drugs}

Flecainide

Amiodarone Ventricular arrhythmias in patients with ventricular conduction in absence of atrioventricular nodal blocking drug torsades de pointes
Bradycardia, heart block, heart failure, constipation, drug interactions

Well tolerated with levels in the therapeutic range. Toxicity manifest with gastrointestinal disturbance, visual disturbance, heart block and ventricular arrhythmias
Contraindications

High-grade atrioventricular block, bronchospastic asthma, decompensated heart failure High-grade atrioventricular block, decompensated heart failure

Severe renal dysfunction (relative), high-grade atrioventricular block

Left ventricular dysfunction, coronary structural heart disease. Atrial flutter with 1:1 Bradycardia, heart block, QT prolongation,

Thyrotoxicosis, sleep disturbance, cutaneous photosensitivity, tremor. Pulmonary fibrosis and hepatitis are rare

Highest risk of torsades de pointes in older women ( $>70$ years) with renal impairment or patients with prolonged corrected QT interval

Thyrotoxicosis, pulmonary fibrosis (relative)

\section{Comments}

Particularly used in setting of coexistent coronary artery disease or left ventricular systolic dysfunction

Not effective for rate control of AF during exercise. Useful in patients with hypotension complicating use of other drugs. Monitor levels to prevent toxicity
Good first choice, in combination with a rate control drug, in patients with paroxysmal AF in absence of structural heart disease

Good first choice in younger patients ( $<65$ years) with paroxysmal AF and preserved renal function

First-line choice in patients with AF and heart failure. Avoid use in younger patients if possible. Monitor thyroid function 3-6-monthly

* The list of adverse effects and contraindications shown here is not exhaustive and the product information for each medication should be consulted before prescribing. 


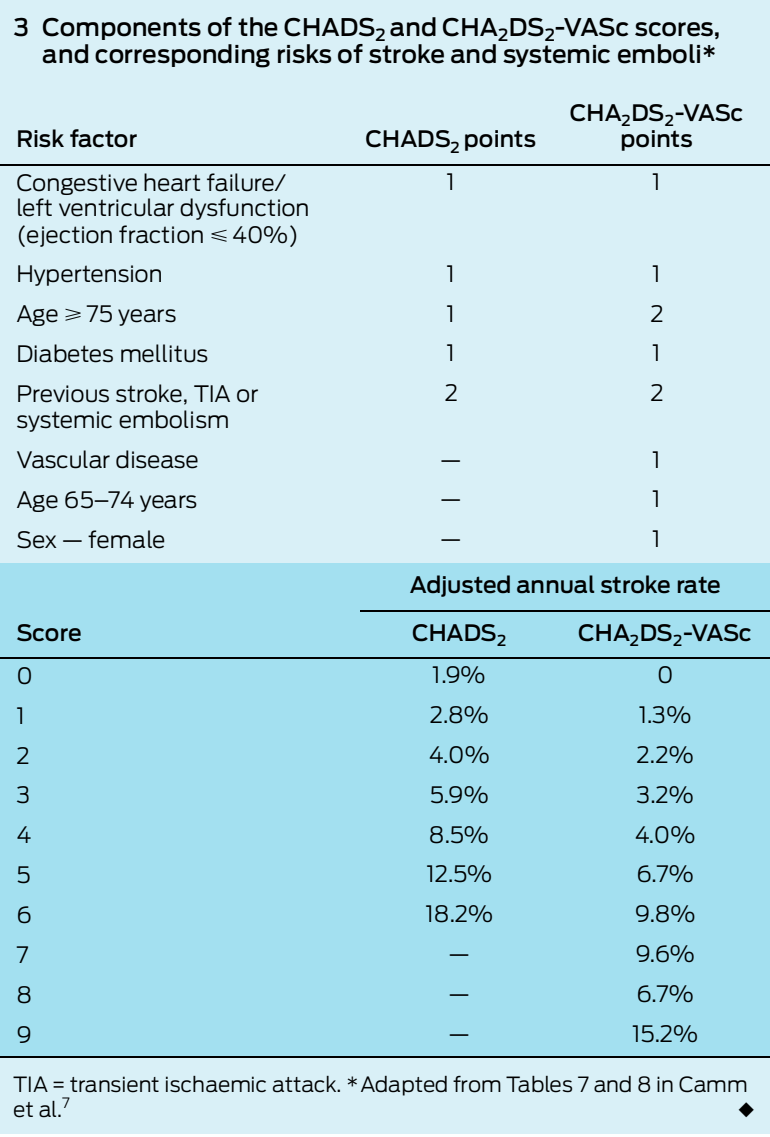

ing electrical properties of the atria. Flecainide, sotalol and amiodarone are the most commonly used in Australia (Box 2). These agents carry the risk of ventricular pro-arrhythmia (flecainide and sotalol) or excessive bradycardia (sotalol and amiodarone), which has been implicated in explaining why studies have failed to show any survival advantage from a rhythm control strategy. Flecainide is contraindicated in patients with left ventricular dysfunction or coronary artery disease due to the risk of ventricular arrhythmias. ${ }^{30}$ As it may also facilitate transition of AF to atrial flutter with 1:1 conduction, leading to haemodynamic collapse, it should be given with an atrioventricular nodal blocking drug. Sotalol is associated with prolongation of the QT interval and pause-dependent polymorphic ventricular tachycardia (torsades de pointes), particularly at higher doses and in older women $(>70$ years) with renal dysfunction, as well as in patients with left ventricular systolic dysfunction and past myocardial infarction. Amiodarone is associated with thyrotoxicosis, sleep disturbance, cutaneous photosensitivity and tremor. Pulmonary fibrosis and hepatitis are rare complications. Its use should be avoided in younger patients if possible.

\section{Catheter ablation}

Catheter ablation is an effective strategy for long-term control of AF in appropriate patients. The recently published National Heart Foundation of Australia consensus statement advises that the primary indication for catheter ablation is symptomatic AF that is refractory to, or in a patient intolerant of, at least one antiarrhythmic drug. ${ }^{31}$ In such patients, AF ablation has the potential to dramatically restore quality of life. A recent study demonstrated no benefit of a primary ablative approach in patients who had not trialled medical therapy, but $36 \%$ of medically treated patients crossed over to ablation during the 2-year followup period. ${ }^{32}$ At present, there is no evidence that $\mathrm{AF}$ ablation reduces risk of stroke or heart failure or improves survival. A large international study examining these questions is ongoing. Appropriate selection of patients is important, with the best results demonstrated in younger patients with paroxysmal or early persistent $\mathrm{AF}$, no advanced structural heart disease and without marked left atrial enlargement. ${ }^{33}$

Randomised controlled trials have reported the success of pulmonary vein isolation in maintaining sinus rhythm to be between $66 \%$ and $89 \%$ at 12 -month follow-up. ${ }^{33}$ In a meta-analysis of patients with paroxysmal or persistent $\mathrm{AF}$, the single-procedure success rate of ablation, with freedom from antiarrhythmic drug therapy, was 57\%, and the multiple-procedure success rate was $71 \% .{ }^{34}$ In a parallel analysis of antiarrhythmic drug therapy, the overall rate of freedom from arrhythmia during the follow-up period was $52 \% .^{34}$ Predictors of AF recurrence after ablation are persistent $\mathrm{AF}$, marked left atrial enlargement and hypertension. It is important to continue to actively control comorbidities such as hypertension, OSA and obesity after ablation. The most common reason for AF recurrence remains recovery of conduction from the pulmonary veins to the left atrium, and about $20 \%-30 \%$ of patients may require repeat ablation for this reason. Studies have now demonstrated cost-effectiveness of ablation compared with ongoing medical therapy for paroxysmal $\mathrm{AF}$, particularly in younger patients. ${ }^{35,36}$

Catheter ablation of $\mathrm{AF}$ is a complex interventional procedure that requires experienced operators and laboratory time of $2-4$ hours on each occasion. There is a $1 \%-2 \%$ risk of major complications, including stroke, cardiac tamponade, and atrio-oesophageal fistula. The mortality risk is estimated at about $0.1 \% .^{33,37}$ The discussion about whether to undergo this procedure is necessarily detailed but it is reasonable to hope that, with ongoing evolution of ablation technologies and an improving understanding of the mechanisms underlying persistent $\mathrm{AF}$, procedural success rates will continue to improve, with reduced risk.

\section{Stroke prevention}

Ischaemic stroke, the risk of which is defined by clearly identified risk factors (Box 3), occurs in about $5 \%$ of patients with non-valvular AF annually and is its most feared complication. Until recently, warfarin and antiplatelet agents were the only treatments available for stroke reduction. Aspirin confers a 19\% non-significant reduction in the relative risk of stroke compared with placebo, while adjusted-dose warfarin (international normalised ratio [INR], 2.0-3.0) significantly reduces the risk of stroke by $64 \% .^{38,39}$ In head-to-head trials against either aspirin or aspirin plus clopidogrel, warfarin was significantly more effective for stroke reduction, with a relative risk reduction of $38 \%$ when compared with aspirin and $42 \%$ when compared with dual antiplatelet therapy. ${ }^{38,40-42}$ 
4 Components of the HAS-BLED score and corresponding risk of bleeding in 3071 patients with atrial fibrillation*

\section{Risk factor}

HAS-BLED points

\begin{tabular}{lc} 
Hypertension & 1 \\
Abnormal renal and/or liver function & 1 or 2 \\
Stroke & 1 \\
Bleeding & 1 \\
Labile international normalised ratio & 1 \\
Elderly (age $>$ 65 years) & 1 or 2 \\
Drug therapy ${ }^{\dagger}$ and/or alcohol misuse & Bleeds per 100 patient-years \\
HAS-BLED score (number of patients) & 1.13 \\
\hline (798) & 1.02 \\
$1(1286)$ & 1.88 \\
$2(744)$ & 3.74 \\
$3(187)$ & 8.70 \\
$4(46)$ & 12.50 \\
$5(8)$ & 0 \\
$6(2)$ & - \\
$7(0)$ & - \\
$8(0)$ & - \\
$9(0)$ &
\end{tabular}

*Adapted from Tables 2 and 5 in Pisters et al. ${ }^{47}+$ Such as aspirin, non-steroidal anti-inflammatory drugs or steroids.

Despite these data, many patients who would benefit from systemic anticoagulation are not taking warfarin. Patients may be reluctant to take warfarin because of anecdotal reports of bleeding or apprehension about the requirement for regular blood tests and restrictions in food and alcohol consumption. Physician factors involved with underprescription of anticoagulation include a perception that the stroke risk is low or that the bleeding risk is excessive, with many doctors perhaps more influenced by the perceived bleeding risk than the benefits of stroke reduction. Given that stroke related to AF often necessitates hospitalisation, carries a significant risk of mortality and is frequently disabling with a need for long-term institutional care, ${ }^{43,44}$ it is important that the risks and benefits of systemic anticoagulation are accurately appreciated.

\section{Balancing the risks of stroke and bleeding}

The most commonly accepted risk factors for stroke in non-valvular AF are a history of thromboembolism, heart failure, hypertension or diabetes and age $\geqslant 75$ years, ${ }^{45}$ as described in the most commonly used risk stratification model, the $\mathrm{CHADS}_{2}$ score (Box 3). This reliably identifies patients at intermediate and high risk of stroke but less reliably identifies those truly at low risk. ${ }^{46}$ Anticoagulation is recommended if the $\mathrm{CHADS}_{2}$ score is $\geqslant 2$ and should be considered if the score is 1 . To refine this scoring system, the $\mathrm{CHA}_{2} \mathrm{DS}_{2}$-VASc score has been developed - in addition to the components of the $\mathrm{CHADS}_{2}$ score, it includes age 65-74 years, vascular disease and female sex as risk factors. The European Society of Cardiology's AF management guidelines propose a lower threshold for anticoagulation and recommend its use if the $\mathrm{CHA}_{2} \mathrm{DS}_{2}$-VASc score is $\geqslant 1$, with the emphasis changed from asking which patients with non-valvular AF should receive anticoagulation to which patients should not. ${ }^{7}$ The risk of stroke across the clinical spectrum of AF is governed by the same set of risk factors, which should be used, rather than the type of $\mathrm{AF}$ the patient has, to guide the need for anticoagulation in all patients.

Physicians are understandably concerned about the bleeding risk of anticoagulation in their patients and have traditionally evaluated bleeding risk intuitively. The HASBLED score, derived from 3456 patients with AF, 53 of whom had major bleeds over 1 year of follow-up, allows a more systematic means of evaluating risk (Box 4). ${ }^{47} \mathrm{How}^{-}$ ever, a high HAS-BLED score $(\geqslant 3)$ should not preclude anticoagulation, as these are often the patients with the highest risk of stroke, who have the most to gain from anticoagulation. ${ }^{48}$ Indeed, a recent large registry study has shown that patients with high bleeding risk still benefit from anticoagulation. ${ }^{49}$ An important function of the HAS-BLED score is to identify potentially reversible risk factors with a view to intervention; for example, discontinuation of aspirin and control of hypertension. It is worth emphasising that adding antiplatelet therapy to any form of anticoagulation increases the risk of bleeding without affecting efficacy. ${ }^{50}$

\section{New oral anticoagulants}

NOAC drugs have been developed as alternatives to warfarin. The three agents proven in clinical trials are the factor Xa inhibitors rivaroxaban and apixaban, and the direct thrombin inhibitor dabigatran. These all have a rapid onset of action, with full anticoagulation 2-3 hours after oral administration; provide predictable anticoagulation so that there is no need for monitoring; and have few food and drug interactions. If renal function is normal, given the relatively short half-life and pharmacokinetics of the NOACs, coagulation should return to normal 24-48 hours after discontinuation. This normalisation is prolonged in the setting of renal dysfunction, particularly in the case of dabigatran, which is $80 \%$ cleared renally. Rivaroxaban and apixaban are partially metabolised by the cytochrome P450 system and have potential for interaction with drugs such as azole antifungals and macrolide antibiotics. Dabigatran is a substrate for the P-glycoprotein system and has potential for interaction with azole antifungals, rifampicin, verapamil and amiodarone.

5 Risks of stroke or systemic embolism and of intracranial bleeding for the new oral anticoagulant drugs when compared with warfarin ${ }^{51-53}$

Stroke or systemic embolism

\begin{tabular}{|c|c|c|c|c|}
\hline \multirow[b]{2}{*}{ Drug } & & & \\
\hline & Relative risk (95\% Cl) & Hazard ratio $(95 \% \mathrm{Cl})$ & Relative risk ( $95 \% \mathrm{CI}$ ) & Hazard ratio $(95 \% \mathrm{Cl})$ \\
\hline Dabigatran 110 mg & $0.91(0.74-1.11)$ & & $0.31(0.20-0.47)$ & \\
\hline Dabigatran 150 mg & $0.66(0.53-0.82)$ & & $0.40(0.27-0.60)$ & \\
\hline Rivaroxaban & & 0.88 (0.75-1.03) & & 0.67 (0.47-0.93) \\
\hline Apixaban & & $0.79(0.66-0.95)$ & & $0.42(0.30-0.58)$ \\
\hline
\end{tabular}




\section{Clinical case study}

A 60-year-old woman complains of intermittent palpitations, with episodes lasting for hours before spontaneously resolving, but becoming more frequent and of longer duration. She becomes fatigued during these episodes but has continued to carry out her usual activities. She has well controlled type 2 diabetes and well controlled hypertension. She experienced a myocardial infarction 3 years ago.

She is in sinus rhythm when she presents, with an electrocardiogram demonstrating inferior Q-waves, but a Holter monitor confirms paroxysmal atrial fibrillation (AF). A subsequent echocardiogram shows normal left ventricular size and preserved systolic function, with mild inferior wall hypokinesis, mild left atrial dilatation and normal valve function. Results of thyroid function and biochemistry tests are normal.

The central management issues for this patient are control of her symptoms (palpitations and fatigue) and reduction of her significant risk of ischaemic stroke.

As the patient has symptomatic paroxysmal $\mathrm{AF}$, with a $\mathrm{CHADS}_{2}$ score of 2 and a $\mathrm{CHA}_{2} \mathrm{DS}_{2-}$ VASc score of 4 , she should receive anticoagulation. Given her symptoms associated with episodes of AF, an initial rhythm control approach with sotalol would be reasonable. Catheter ablation should then be considered if a trial of medical therapy fails to control her symptoms: the presence of only mild atrial enlargement confers a good chance of sustained freedom from AF. However, given her high risk score, anticoagulation should be continued even if she appears to be in long-term sinus rhythm.

Trials have shown that all three drugs are at least as effective as warfarin in reducing stroke risk in patients with non-valvular $\mathrm{AF}$, and all have significantly lower rates of intracranial haemorrhage than warfarin (Box 5). ${ }^{51-53} \mathrm{~A}$ significant reduction in major bleeding was found with $110 \mathrm{mg}$ of dabigatran and with apixaban. The $150 \mathrm{mg}$ dose of dabigatran was associated with an excess of major (largely gastrointestinal) bleeding in those aged $\geqslant 75$ years. ${ }^{54}$ but significantly reduced ischaemic stroke. Rivaroxaban had a similar rate of major bleeding as warfarin, but an excess of major gastrointestinal bleeding. The lower dose of dabigatran (110 mg twice daily) should be used in patients aged $\geqslant 75$ years, and it should be considered in patients with moderate renal dysfunction (creatinine clearance, $30-50 \mathrm{~mL} / \mathrm{min}$ ) and high bleeding risk. A lower dose of rivaroxaban $(15 \mathrm{mg})$ should be used in patients with moderate renal dysfunction (creatinine clearance, 30$50 \mathrm{~mL} / \mathrm{min}$ ). Dose reduction with apixaban (to $2.5 \mathrm{mg}$ twice daily) is indicated when patients have two of the following high-risk features: bodyweight $<60 \mathrm{~kg}$, serum creatinine level $>133 \mu \mathrm{mol} / \mathrm{L}$, and age $\geqslant 80$ years.

A concern specific to NOACs is the management of intercurrent bleeding. While warfarin can be slowly reversed with vitamin $\mathrm{K}$ and clotting factors, there are currently no specific antidotes for NOACs available. Prothrombin complex concentrates have been shown to normalise tests of coagulation in healthy controls taking rivaroxaban, but there have been no studies of the ability of such concentrates to stop pathological bleeding. A neutralising monoclonal antibody to inhibit dabigatran is in evaluation. If bleeding does occur, supportive measures may be sufficient in many instances until drug levels decline and coagulation returns to normal. Maintaining good renal blood flow to promote excretion is important. Haemodialysis can be used to remove dabigatran but is not effective for rivaroxaban or apixaban.

Patients who have not received anticoagulation may form the largest group in whom NOACs will be used, as in all the major studies there was no differential efficacy or safety response according to previous warfarin exposure. ${ }^{51-53}$ Patients with unstable INRs will be targets for a transition. However, attention should first be given to the cause of instability, as a patient who is poorly compliant with warfarin may be equally poorly compliant with NOACs, and this may be more dangerous given their short halflives. Whether patients with stable INR control should be transitioned is a more difficult question. All three major studies have shown that the benefits of the new agents are independent of centre-based time in the therapeutic range. In the Randomized Evaluation of Long-Term Anticoagulation Therapy (RE-LY) trial of dabigatran, there was a marked reduction in intracranial haemorrhage even in patients with good INR control. ${ }^{51}$ Therefore, we feel it is not unreasonable to swap patients with stable INRs to the new agents. This is particularly so for a patient who requests such a change for reasons of convenience, as long as factors such as renal function and drug interactions are considered. However, we do not think there is enough evidence to recommend wholesale transfer of patients with stable INRs to the new agents.

All the NOACs have a higher cost than warfarin. Nevertheless, the reduction in stroke, bleeding and need for monitoring and physician consultation may more than counterbalance this, and cost-benefit analyses in favour of dabigatran have been published. ${ }^{55}$

\section{Summary}

$\mathrm{AF}$ is a common and increasingly prevalent condition that may be associated with a wide spectrum of symptom severity. Whether a patient is symptomatic or not, reducing stroke risk remains a central consideration. Key management decisions are whether to adopt a rhythm control or rate control strategy and whether to initiate anticoagulation (Box 6). Rhythm control with antiarrhythmic therapy is primarily aimed at symptom control. Ablation should be considered in younger patients, particularly if they have paroxysmal AF and no more than mild left atrial enlargement. Anticoagulation should be considered based on stroke risk assessed using the $\mathrm{CHADS}_{2}$ or $\mathrm{CHA}_{2} \mathrm{DS}_{2}$-VASc scoring system, rather than on the type of AF. It is likely that the NOAC drugs will be increasingly used for this indication but, as with any new therapy, it is important to be prudent with their use while clinical experience is building. Given the proven efficacy of warfarin for stroke reduction, a high degree of vigilance for potential adverse effects from NOACs is required.

Acknowledgements: Tomos Walters is supported by a postgraduate scholarship cofounded by the National Health and Medical Research Council and the National Heart Foundation.

Competing interests: John Amerena was on the steering committee and was national coordinator/principal investigator for the RE-LY and ARISTOTLE studies and was a participant in the ROCKET AF study. He has been on national and international advisory boards for the manufacturers of all three novel anticoagulants and has given sponsored and invited lectures nationally and internationally. Jonathan Kalman has received investigator-initiated research and fellowship support from St Jude Medical, Medtronic, Biosense Webster and Boston Scientific.

Provenance: Commissioned; externally peer reviewed.

1 Go AS, Hylek EM, Phillips KA, et al. Prevalence of diagnosed atrial fibrillation in adults: national implications for rhythm management and stroke prevention: the AnTicoagulation and Risk Factors in Atrial Fibrillation (ATRIA) Study. JAMA 2001; 285: 2370-2375.

2 Lloyd-Jones DM, Wang TJ, Leip EP, et al. Lifetime risk for development of atrial fibrillation: the Framingham Heart Study. Circulation 2004; 110: 1042-1046.

3 Heeringa J, van der Kuip DAM, Hofman A, et al. Prevalence, incidence and lifetime risk of atrial fibrillation: the Rotterdam study. Eur Heart J 2006; 27 : 949-953. 
4 Benjamin EJ, Chen P-S, Bild DE, et al. Prevention of atrial fibrillation: report from a National Heart, Lung, and Blood Institute workshop. Circulation 2009; 119: 606-618.

5 Steger C, Pratter A, Martinek-Bregel M, et al. Stroke patients with atrial fibrillation have a worse prognosis than patients without: data from the Austrian Stroke registry. Eur Heart J 2004; 25: 1734-1740.

6 Lip GYH, Tse HF, Lane DA. Atrial fibrillation. Lancet 2012; 379: 648-661.

7 Camm AJ, Kirchhof P, Lip GYH, et al. Guidelines for the management of atrial fibrillation: the Task Force for the Management of Atrial Fibrillation of the European Society of Cardiology (ESC). Eur Heart J 2010; 31: 2369-2429.

8 Kumar S, Walters TE, Halloran K, et al. Ten-year trends in the use of catheter ablation for treatment of atrial fibrillation vs. the use of coronary intervention for the treatment of ischaemic heart disease in Australia. Europace 2013; Jun 24 [Epub ahead of print].

9 Benjamin EJ, Levy D, Vaziri SM, et al. Independent risk factors for atrial fibrillation in a population-based cohort. The Framingham Heart Study. JAMA 1994; 271: 840-844.

10 Ryder KM, Benjamin EJ. Epidemiology and significance of atrial fibrillation. Am J Cardiol 1999; 84: 131R-138R.

11 Wang TJ, Parise H, Levy D, et al. Obesity and the risk of new-onset atrial fibrillation. JAMA 2004; 292: 2471-2477.

12 Frost L, Hune LJ, Vestergaard P. Overweight and obesity as risk factors for atrial fibrillation or flutter: the Danish Diet, Cancer, and Health Study. Am J Med 2005; 118: 489-495.

13 Stewart S, Hart CL, Hole DJ, McMurray JJ. Population prevalence, incidence, and predictors of atrial fibrillation in the Renfrew/Paisley study. Heart 2001; 86: 516-521.

14 Tedrow UB, Conen D, Ridker PM, et al. The long- and short-term impact of elevated body mass index on the risk of new atrial fibrillation: the WHS (Women's Health Study). J Am Coll Cardiol 2010; 55: 2319-2327.

15 Thanassoulis G, Massaro JM, O'Donnell CJ, et al. Pericardial fat is associated with prevalent atrial fibrillation: the Framingham Heart Study. Circ Arrhythm Electrophysiol 2010; 3: 345-350.

16 Wong CX, Abed HS, Molaee P, et al. Pericardial fat is associated with atrial fibrillation severity and ablation outcome. J Am Coll Cardiol 2011; 57: 1745-1751.

17 Gami AS, Pressman G, Caples SM, et al. Association of atrial fibrillation and obstructive sleep apnea. Circulation 2004; 110: 364-367.

18 Gami AS, Hodge DO, Herges RM, et al. Obstructive sleep apnea, obesity, and the risk of incident atrial fibrillation. J Am Coll Cardiol 2007; 49: 565-571.

19 Stevenson IH, Teichtahl H, Cunnington D, et al. Prevalence of sleep disordered breathing in paroxysmal and persistent atrial fibrillation patients with normal left ventricular function. Eur Heart J 2008; 29: 1662-1669.

20 Huxley RR, Lopez FL, Folsom AR, et al. Absolute and attributable risks of atrial fibrillation in relation to optimal and borderline risk factors: the Atherosclerosis Risk in Communities (ARIC) study. Circulation 2011; 123: 1501-1508.

21 Potpara TS, Lip GYH. Lone atrial fibrillation: what is known and what is to come. Int J Clin Pract 2011; 65: 446-457.

22 Hohnloser SH, Kuck KH, Lilienthal J. Rhythm or rate control in atrial fibrillation - Pharmacological Intervention in Atrial Fibrillation (PIAF): a randomised trial. Lancet 2000; 356: 1789-1794.

23 Wyse DG, Waldo AL, DiMarco JP, et al. A comparison of rate control and rhythm control in patients with atrial fibrillation. N Engl J Med 2002; 347: 1825-1833.

24 Van Gelder IC, Hagens VE, Bosker HA, et al. A comparison of rate control and rhythm control in patients with recurrent persistent atrial fibrillation. $\mathrm{N}$ Engl $\mathrm{J}$ Med 2002; 347: 1834-1840.

25 Carlsson J, Miketic S, Windeler J, et al. Randomized trial of rate-control versus rhythm-control in persistent atrial fibrillation: the Strategies of Treatment of Atrial Fibrillation (STAF) study. J Am Coll Cardiol 2003; 41: 1690-1696.

26 Ogawa S, Yamashita T, Yamazaki T, et al. Optimal treatment strategy for patients with paroxysmal atrial fibrillation: J-RHYTHM Study. Circ J 2009; 73: 242-248.

27 Roy D, Talajic M, Nattel S, et al. Rhythm control versus rate control for atrial fibrillation and heart failure. N Engl J Med 2008; 358: 2667-2677.

28 Caldeira D, David C, Sampaio C. Rate versus rhythm control in atrial fibrillation and clinical outcomes: updated systematic review and meta-analysis of randomized controlled trials. Arch Cardiovasc Dis 2012; 105: 226-238.

29 Van Gelder IC, Groenveld HF, Crijns HJGM, et al. Lenient versus strict rate control in patients with atrial fibrillation. N Engl J Med 2010; 362: 1363-1373.

30 Echt DS, Liebson PR, Mitchell LB, et al. Mortality and morbidity in patients receiving encainide, flecainide, or placebo - The Cardiac Arrhythmia Suppression Trial. NEngl J Med 1991; 324: 781-788.

31 Kalman JM, Sanders P, Brieger DB, et al. National Heart Foundation of Australia consensus statement on catheter ablation as a therapy for atrial fibrillation. Med J Aust 2013; 198: 27-28.

32 Cosedis Nielsen J, Johannessen A, Raatikainen P, et al. Radiofrequency ablation as initial therapy in paroxysmal atrial fibrillation. NEngl J Med 2012; 367: 1587-1595.
33 Calkins H, Kuck K-H, Cappato R, et al. 2012 HRS/EHRA/ECAS Expert consensus statement on catheter and surgical ablation of atrial fibrillation: recommendations for patient selection, procedural techniques, patient management and follow-up, definitions, endpoints, and research trial design. Europace 2012; 14: 528-606.

34 Calkins H, Reynolds MR, Spector P, et al. Treatment of atrial fibrillation with antiarrhythmic drugs or radiofrequency ablation: two systematic literature reviews and meta-analyses. Circ Arrhythm Electrophysiol 2009; 2: 349-361.

35 Weerasooriya R, Jaïs P, Le Heuzey J-Y, et al. Cost analysis of catheter ablation for paroxysmal atrial fibrillation. Pacing Clin Electrophysiol 2003; 26: 292-294.

36 Goldberg A, Menen M, Mickelsen S, et al. Atrial fibrillation ablation leads to long-term improvement of quality of life and reduced utilization of healthcare resources. J Interv Card Electrophysiol 2003; 8: 59-64.

37 Wann LS, Curtis AB, January CT, et al. 2011 ACCF/AHA/HRS focused update on the management of patients with atrial fibrillation (updating the 2006 guideline): a report of the American College of Cardiology Foundation/ American Heart Association Task Force on Practice Guidelines. Circulation 2011; 123: 104-123.

38 Hart RG, Pearce LA, Aguilar MI. Meta-analysis: antithrombotic therapy to prevent stroke in patients who have nonvalvular atrial fibrillation. Ann Intern Med 2007; 146: 857-867.

39 Ansell J, Hirsh J, Hylek E, et al. Pharmacology and management of the vitamin K antagonists: American College of Chest Physicians Evidence-Based Clinical Practice Guidelines (8th Edition). Chest 2008; 133 (6 Suppl): 160S-198S.

40 Mant J, Hobbs FDR, Fletcher K, et al. Warfarin versus aspirin for stroke prevention in an elderly community population with atrial fibrillation (the Birmingham Atrial Fibrillation Treatment of the Aged Study, BAFTA): a randomised controlled trial. Lancet 2007; 370: 493-503.

41 Connolly S, Pogue J, Hart R, et al. Clopidogrel plus aspirin versus oral anticoagulation for atrial fibrillation in the Atrial fibrillation Clopidogrel Trial with Irbesartan for prevention of Vascular Events (ACTIVE W): a randomised controlled trial. Lancet 2006; 367: 1903-1912.

42 Connolly SJ, Pogue J, Eikelboom J, et al. Benefit of oral anticoagulant over antiplatelet therapy in atrial fibrillation depends on the quality of international normalized ratio control achieved by centers and countries as measured by time in therapeutic range. Circulation 2008; 118: 2029-2037.

43 Benjamin EJ, Wolf PA, D'Agostino RB, et al. Impact of atrial fibrillation on the risk of death: the Framingham Heart Study. Circulation 1998; 98: 946-952.

44 Savelieva I, Bajpai A, Camm AJ. Stroke in atrial fibrillation: update on pathophysiology, new antithrombotic therapies, and evolution of procedures and devices. Ann Med 2007; 39: 371-391.

45 Stroke Risk in Atrial Fibrillation Working Group. Independent predictors of stroke in patients with atrial fibrillation: a systematic review. Neurology 2007; 69: 546-554.

46 Olesen JB, Torp-Pedersen C, Hansen ML, Lip GYH. The value of the CHA2DS2VASc score for refining stroke risk stratification in patients with atrial fibrillation with a CHADS2 score 0-1: a nationwide cohort study. Thromb Haemost 2012; 107: 1172-1179.

47 Pisters R, Lane DA, Nieuwlaat R, et al. A novel user-friendly score (HAS-BLED) to assess 1-year risk of major bleeding in patients with atrial fibrillation: the Euro Heart Survey. Chest 2010; 138: 1093-1100.

48 Fauchier L, Lip GYH. Guidelines for antithrombotic therapy in atrial fibrillation: understanding the reasons for non-adherence and moving forwards with simplifying risk stratification for stroke and bleeding. Europace 2010; 12: 761-763.

49 Friberg L, Rosenqvist M, Lip GYH. Net clinical benefit of warfarin in patients with atrial fibrillation: a report from the Swedish atrial fibrillation cohort study. Circulation 2012; 125: 2298-2307.

50 Hansen ML, Sørensen R, Clausen MT, et al. Risk of bleeding with single, dual, or triple therapy with warfarin, aspirin, and clopidogrel in patients with atrial fibrillation. Arch Intern Med 2010; 170: 1433-1441.

51 Connolly SJ, Ezekowitz MD, Yusuf S, et al; RE-LY Steering Committee and Investigators. Dabigatran versus warfarin in patients with atrial fibrillation. NEngl J Med 2009; 361: 1139-1151.

52 Patel MR, Mahaffey KW, Garg J, et al; ROCKET AF Investigators. Rivaroxaban versus warfarin in nonvalvular atrial fibrillation. NEngl J Med 2011; 365: 883891.

53 Granger CB, Alexander JH, McMurray JJV, et al; ARISTOTLE Committees and Investigators. Apixaban versus warfarin in patients with atrial fibrillation. NEngl J Med 2011; 365: 981-992.

54 Eikelboom JW, Wallentin L, Connolly SJ, et al. Risk of bleeding with 2 doses of dabigatran compared with warfarin in older and younger patients with atrial fibrillation: an analysis of the Randomized Evaluation of Long-Term Anticoagulant Therapy (RE-LY) trial. Circulation 2011; 123: 2363-2372. doi: 10.1161/CIRCULATIONAHA.110.004747.

55 Freeman JV, Zhu RP, Owens DK, et al. Cost-effectiveness of dabigatran compared with warfarin for stroke prevention in atrial fibrillation. Ann Intern Med 2011; 154: 1-11. 